\title{
The Prevalence and Incidence of Dementia in Elderly Urban Japanese : The Sendai Longitudinal Study of Aging
}

\author{
Yuko Minami ${ }^{1}$, Ichiro Tsuji ${ }^{1,2}$, Penelope M. Keyl ${ }^{2}$, Shigeru Hisamichi ${ }^{1}$, \\ Hirotake Asano ${ }^{3}$, Makito Sato ${ }^{3}$, and Kazuo Shinoda ${ }^{4}$
}

\begin{abstract}
To investigate the age-associated changes in physical and mental functions, epidemiological surveys were conducted for a random sample $(N=3,704)$ of residents aged 65 years and over in Sendai City, Japan, in 1988 and 1991. The frequency of dementia was derived from the epidemiological surveys. The prevalence of dementia aged 65 years and over in 1988 was 5.13 per 100 persons with age-specific rates of $0.78,1.89,6.02,11.95$ and 26.31 per 100 persons for the age groups $65-69,70-74,75-79,80-84$, and $85+$, respectively. The incidence during 1988-1991 was 1.74 per 100 person-years with age-specific rates of $0.73,0.87,2.29,3.62$, and 8.48 per 100 person-years, respectively. Using the Japan population in 1985, the agestandardized rates for females were higher than those for males for both prevalence and incidence. The prevalence in this study was consistent with those of other population-based studies in urban Japan. There are few studies of the incidence of dementia in Japan, and this is the first such study based on a longitudinal design in an urban area of Japan. J Epidemiol, $1993 ; 3: 83-89$.
\end{abstract}

aged, dementia, incidence, longitudinal studies, prevalence

Population projections indicate that the Japanese population is getting older at the highest in the world ${ }^{1,2)}$. The rapid increase in the number of the elderly will create additional pressure on families and public health services ${ }^{3,4)}$. Dementia will become a major public health problem since it occurs among the elderly very often and affects the ability of people to live independently ${ }^{5}$. In order to provide effective and efficient health services for the demented elderly, it is crucial to understand the natural course of dementia. So far, various cross-sectional studies on dementia have been conducted to ascertain prevalence and service needs for dementia ${ }^{6-19}$. However, information on the incidence and the natural course of dementia $^{19-28)}$ is limited. The elucidation of such information, which can be obtained by longitudinal studies, would be useful not only for planning health services but also for preventing the progression of physical and mental disorders. The need for longitudinal studies on the aging population has been addressed in recent years $^{29-31)}$.

The aim of the Sendai Longitudinal Study of Aging is to investigate the age-associated changes in physical and mental functions among the elderly through a population-based epidemiological study. This paper describes the study design and the estimates of prevalence and incidence of dementia in this population. Although studies of the prevalence have often been carried out in Japan ${ }^{5-13)}$, there are few studies of the incidence of dementia in Japan ${ }^{20-22)}$. This is the first such study based on a longitudinal design in an urban area of Japan.

\section{MATERIALS AND METHODS}

\section{Study design}

Sendai is the largest city in the northeastern area of "Honshu (Main)" Island, Japan. Its population in

\footnotetext{
Received April 20, 1993; accepted August 20, 1993.

'Department of Public Health, Tohoku University School of Medicine, Sendai, Japan. ${ }^{2}$ Department of Epidemiology, Johns Hopkins University School of Hygiene and Public Health, Baltimore, MD, USA. '3Sendai City Health Department, Sendai, Japan.

"Department of Psychiatry, Tohoku-Kai Hospital, Sendai, Japan.

Address for correspondence: Yuko Minami, Department of Public Health, Tohoku University School of Medicine, 2-1 Seiryo-machi Aoba-ku Sendai, 980, Japan.
} 
1988 was approximately 880,000 , of whom $8.4 \%$ were aged 65 and over. On the 1st October, 1988, a study sample of 3,704 subjects ( $5 \%$ of residents aged 65 and over) was randomly selected from the residents' registration cards maintained by the city. Sampling was stratified by each year of age.

This population-based study consists of two surveys taken three years apart, Wave I Study taken during October-December, 1988 and Wave II Study performed during October-December 1991. Just before starting Wave II Study, we obtained information from the Sendai City Government office on the present status (deceased, moved away, or still living in Sendai City) of respondents in Wave I. For the decedents, the causes of death were obtained from death certificates. Wave II Study was conducted for the survivors still living in Sendai to investigate the age-associated changes in physical and mental function. Information on subjects who left Sendai City was not obtained.

Each Wave consisted of an identical 2-stage investigation. In Stage 1 Survey, a member of regional welfare committee visited each subject at his or her own residence and asked him or her to complete a questionnaire about demographic data, self-rated health status, physical disability, mental health status, and previously diagnosed diseases. When the subject selected was not able to respond, a proxy was asked to complete the questionnaire. But, it was requested that the evaluation of mental health status, which was necessary for screening suspected dementia, should be carried out by only a proxy. A few days later, the same person collected the completed questionnaire.

From the responses to this questionnaire, subjects were classified as not demented or as dementia suspected, using the screening criteria shown in Table 1 , which was established by a committee of seven psychiatrists. All those in the latter category were invited to
Stage 2, consisting of interview and examination. In Stage 2, trained public health nurses visited the suspected dementia subjects individually, interviewing and giving physical and psychiatric examinations including the examinations of Karasawa's senility scale ${ }^{6,32)}$ and Hasegawa's dementia scale ${ }^{32,33)}$. Karasawa's senility scale is an instrument to evaluate severity of intellectual deterioration according to an interviewer's judgement. Hasegawa's dementia scale is widely used as the standard screening instrument in Japan, similar to the Mini-Mental State Examination ${ }^{34)}$. At the same time, information about the level of care and service needs were obtained from caregivers.

Based on the information collected by nurses, the diagnostic evaluation of dementia was made by the seven member-psychiatric committee, taking the Diagnostic and Statistical Manual of Mental Disorders, Third Edition-Revised (DSM-III-R) ${ }^{35)}$, Karasawa's senility scale and Hasegawa's dementia scale into consideration. The subjects who were difficult to clearly diagnose as dementia or non-dementia using the information collected by the nurses were given direct physical and psychiatric examinations by a member of the committee. Differentiation by type of dementia was not attempted, for the information obtained in Stage 2 was not sufficient to make this determination with confidence.

\section{Prevalence of dementia}

The prevalence rates of dementia. were estimated from Wave I. Out of the 3,704 selected sample, 3,459 subjects $(93.4 \%)$ responded to Stage 1 Survey in Wave I. Of the respondents, 3,376 subjects lived at home and 83 subjects stayed at hospitals or long-term care facilities. Age and sex distribution of the study subjects are shown in Table 2. There was no significant difference in participation rates among sex and five-

Table 1. Screening criteria for suspected dementia in Sendai longitudinal study of aging, 1988-1991.

A subject satisfying at least one of the following twelve items is categorized as suspected dementia.

(I) Often forgets important things or requests

(2) Cries for no obvious reason at night

(3) Turns night into day

(4) Loses his way home

(5) Forgets the names of family members

(6) Forgets to turn off the heating and cooking equipment

(7) Has developed character change

(8) Wanders about

(9) Dirities himself

(10) Sometimes forgets important things or requests/and has a past history of cerebrovascular disease

(11) Sometimes forgets important things or requests/and has a past history of head trauma

(12) Has a past history of depression 
Table 2. Distribution of study subjects by sex and age in wave I, Sendai city, Japan, 1988.

\begin{tabular}{lrccccc}
\hline \multirow{2}{*}{$\begin{array}{c}\text { Age } \\
\text { in } 1988 \\
\text { (years) }\end{array}$} & \multicolumn{3}{c}{ Selected sample } & \multicolumn{4}{c}{ Respondents } \\
\cline { 2 - 7 } & Males & Females & Total & Males & Females & Total \\
\hline $65-69$ & 551 & 756 & 1,307 & $524(5)^{*}$ & $701(10)$ & $1,225(15)$ \\
$70-74$ & 435 & 575 & 1,010 & $408(6)$ & $536(10)$ & $944(16)$ \\
$75-79$ & 290 & 434 & 724 & $279(8)$ & $400(13)$ & $679(21)$ \\
$80-84$ & 152 & 228 & 380 & $143(4)$ & $215(8)$ & $358(12)$ \\
$85-$ & 91 & 192 & 283 & $90(4)$ & $163(15)$ & $253(19)$ \\
Total & 1,519 & 2,185 & 3.704 & $1,444(27)$ & $2,015(56)$ & $3,459(83)$ \\
\hline
\end{tabular}

* : Figures in parentheses indicate the number of subjects staying at hospitals or long-term care facilities

year age groups. Of 3,459 respondents to Stage 1 Survey, 444 subjects were categorized as dementia suspected using the screening criteria. Of those, 363 subjects participated in Stage 2 . Overall and age-, sex-specific prevalence rates of dementia were estimated from Wave I data, based on the assumption that the rate of dementia in Stage 2 was the same for those invited to Stage 2 who did and did not participate in that stage. It was also assumed that no cases of dementia were missed by Stage 1. The following equation was used to estimate prevalence at Wave I :

Prevalence rate $($ per 100 persons $)=\frac{\frac{d}{n 2} \times s}{n 1} \times 100$

where,

$n \mathbf{l}=$ number of subjects who participated in Stage 1

$s=$ number of subjects who were invited to Stage 2

$n 2=$ number of subjects who participated in Stage 2

$\mathrm{d}=$ number of subjects who were diagnosed with dementia at Stage 2

\section{Incidence of dementia}

The incidence rates of dementia for the period 19881991 were estimated using the person-years method. The population at risk comprised all respondents $(\mathrm{N}=$ $3,233)$ with clear evidence of non-dementia in Wave I. That is, those surveyed in 1988, excluding those diagnosed with dementia in 1988 and those with suspected dementia who were not evaluated at Stage 2 in 1988. The status of the population at risk at the time of Wave II by age in 1988 is shown in Table 3. In the estimation of incidence rates, we used data on the at risk population who lived at home in 1988 and who had been followed up ( $\mathrm{N}=2,542)$. The deceased subjects $(N=264)$ and those who had moved out of Sendai City $(N=69)$ were excluded from the analyses. Furthermore, the subjects who had lived at hospitals or long-term care facilities in $1988(\mathrm{~N}=35)$ were also excluded, since the participation rate for them in Wave II was extremely low (14.3\%), in comparison with the participation rate of $88.7 \%$ for subjects who had lived at home.

Person-years were calculated and allocated on the five-year age group, by treating the period of observa-

Table 3. Status at wave II of the population at risk of dementia in 1988, Sendai city, Japan.

\begin{tabular}{|c|c|c|c|c|c|c|c|c|}
\hline \multirow{3}{*}{$\begin{array}{c}\text { Age } \\
\text { in } 1988 \\
\text { (years) }\end{array}$} & \multicolumn{2}{|c|}{ Population at risk } & \multicolumn{6}{|c|}{ Status at Wave II } \\
\hline & \multirow{2}{*}{ at Home } & \multirow{2}{*}{ at $\mathrm{LTC}^{*}$} & \multicolumn{2}{|c|}{ Deceased } & \multicolumn{2}{|c|}{ Moved out } & \multicolumn{2}{|c|}{ Survived } \\
\hline & & & at Home & at $\mathrm{LTC}$ & at Home & at LTC & at Home & at LTC \\
\hline $65-69$ & 1,196 & 11 & 43 & 2 & 20 & 2 & $1,133(1,000)^{\dagger}$ & $7(1)$ \\
\hline $70-74$ & 903 & 14 & 46 & 1 & 17 & 1 & $840 \quad(759)$ & $12(1)$ \\
\hline $75-79$ & 610 & 11 & 53 & 4 & 20 & 0 & $(477)$ & $7(1)$ \\
\hline $80-84$ & 300 & 9 & 51 & 3 & 6 & 0 & $(217)$ & $6(1)$ \\
\hline $85-$ & 171 & 8 & 56 & 5 & 3 & 0 & $122 \quad(89)$ & $3(1)$ \\
\hline Total & 3,180 & 53 & 249 & 15 & 66 & 3 & $1,865 \quad(2,542)$ & $35(5)$ \\
\hline
\end{tabular}

* LTC: Hospitals or long-term care facilities

$\dagger$ : Figures in parentheses indicate the number of subjects who participated in Wave II in 1991 
tion for non-incident subjects as 3 years and for incident subjects as, on average, 1.5 years, since the time of onset for each incident subject was unknown. The same assumptions already described in estimating prevalence in 1988 were used to estimate incidence.

\section{RESULTS}

\section{Prevalence}

Of the 363 subjects who participated in Stage 2 in Wave I, 145 were diagnosed with dementia. The estimated prevalence rates by five-year age group and sex, and the estimated overall prevalence rates are presented in Table 4. Age-standardized rates calculated using the Japan population aged 65 and over in 1985 as the standard ${ }^{36)}$ are also presented in Table 4.

The crude prevalence rate was 5.13 per 100 persons and the age-standardized rate was 4.93 per 100 persons. The age-standardized prevalence rate for females (5.41 per 100 persons) was slightly higher than that for males (4.05 per 100 persons). The age-specific prevalence rate increased rapidly with aging in both sexes. The age-specific prevalence rate for females was higher than that for males in all age groups except for that from 70 to 74 years and larger differences between sexes were observed in the older age groups. The prevalence rate for the subjects living at home $(\mathrm{N}=3,376)$ were estimated separately (Table 4$)$. The age-standardized prevalence rate for the subjects living at home (4.53 per 100 persons) was slightly lower than that for all subjects (4.93 per 100 persons).

\section{Incidence}

Of the 2,542 subjects followed up for the estimation of incidence, 428 subjects were categorized as dementia suspected in Stage 1 in Wave II in 1991. Among them, 347 subjects participated in Stage 2, and 105 were diagnosed with dementia. The incidence rates by age and sex based on the estimated number of incident cases and the estimated person-years are shown in Table 5.

The crude incidence rate was 1.74 per 100 person-

Table 4. Prevalence of dementia by age and sex in Sendai city, Japan, 1988.

\begin{tabular}{|c|c|c|c|c|c|c|}
\hline \multirow{3}{*}{$\begin{array}{c}\text { Age } \\
\text { in } 1988 \\
\text { (years) }\end{array}$} & \multicolumn{6}{|c|}{ Prevalence rate (per 100 persons) } \\
\hline & \multicolumn{3}{|c|}{ All subject } & \multicolumn{3}{|c|}{ Subjects living at home } \\
\hline & Males & Females & Total & Males & Females & Total \\
\hline $65-69$ & 0.67 & 0.87 & 0.78 & 0.63 & 0.83 & 0.74 \\
\hline $70-74$ & 2.53 & 1.25 & 1.89 & 2.06 & 1.32 & 1.70 \\
\hline $75-79$ & 4.24 & 7.19 & 6.02 & 4.04 & 6.09 & 5.23 \\
\hline $80-84$ & 9.15 & 13.78 & 11.95 & 8.71 & 13.86 & 11.80 \\
\hline $85-$ & 20.53 & 29.37 & 26.31 & 20.35 & 25.78 & 23.79 \\
\hline Total & 3.97 & 5.98 & 5.13 & 3.63 & 5.31 & 4.59 \\
\hline ASPR* & 4.05 & 5.41 & 4.93 & 3.81 & 4.99 & 4.53 \\
\hline
\end{tabular}

* ASPR : Age-standardized prevalence rate, using the Japan population in 1985 as standard

Table 5. Incidence of dementia by age and sex in Sendai city, Japan, 1988-1991.

\begin{tabular}{|c|c|c|c|c|c|c|c|c|c|}
\hline \multirow{2}{*}{$\begin{array}{c}\text { Age } \\
\text { group } \\
\text { (years) }\end{array}$} & \multicolumn{3}{|c|}{ Estimated person-years } & \multicolumn{3}{|c|}{$\begin{array}{l}\text { Estimated number of } \\
\text { incident cases }\end{array}$} & \multicolumn{3}{|c|}{$\begin{array}{c}\text { Incidence rate } \\
\text { (per } 100 \text { person-years) }\end{array}$} \\
\hline & Males & Females & Total & Males & Females & Total & Males & Females & Total \\
\hline $65-69$ & $1,077.8$ & $1,398.7$ & $2,476.0$ & 5.4 & 12.2 & 18.0 & 0.50 & 0.87 & 0.73 \\
\hline $70-74$ & 980.3 & $1,347.5$ & $2,327.5$ & 9.1 & 11.0 & 20.3 & 0.93 & 0.82 & 0.87 \\
\hline $75-79$ & 617.8 & 935.9 & $1,553.6$ & 12.8 & 22.8 & 35.6 & 2.07 & 2.44 & 2.29 \\
\hline $80-84$ & 310.4 & 454.3 & 765.2 & 9.7 & 18.4 & 27.7 & 3.13 & 4.05 & 3.62 \\
\hline $85-$ & 106.0 & 204.3 & 311.3 & 7.3 & 19.8 & 26.4 & 6.89 & 9.69 & 8.48 \\
\hline Total & $3,092.8$ & $4,338.8$ & $7,431.7$ & 44.1 & 85.4 & 129.5 & 1.43 & 1.97 & 1.74 \\
\hline ASIR* & & & & & & & 1.61 & 2.06 & 1.87 \\
\hline
\end{tabular}

\footnotetext{
* ASIR: Age-standardized incidence rate, using the Japan population in 1985 as standard
} 
years, and the age-standardized incidence rate was 1.87 per 100 person-years. The age-standardized incidence rate for females ( 2.06 per 100 person-years) was slightly higher than that for males (1.61 per 100 person-years). The trend of the incidence rate by age and sex in the older age groups was similar to that of prevalence, except that the incidence rate for females aged 70-74 was almost the same as that for females aged 65-69.

\section{DISCUSSION}

We have described the design of the Sendai Longitudinal Study of Aging, and the prevalence and incidence of dementia in elderly aged 65 and over in this urban population. Since the study sample was randomly selected from general population and the participation rates in each wave of study were high, the results should accurately represent the characteristics of the elderly in this population. Large-scale longitudinal studies like the present study have never been carried out in an urban area in Japan. The study may provide information to help elucidate the natural history of age-associated changes such as dementia and to plan health services for the elderly.

\section{Prevalence}

Numerous studies on the prevalence of dementia have been carried out in different countries ${ }^{6-19)}$, but methodological differences among the studies make it difficult to directly compare the rate. Most of the Japanese studies used similar, though not identical, methods as we did. The two-stage investigation method is common. Hasegawa's dementia scale, Karasawa's senility scale and DSM-III-R are widely used. Although screening criteria in Stage 1 were different among the studies, symptoms related to dementia were assessed by each study. Since our criteria were so broad and comprehensive, it is unlikely that a substantial number of demented subjects were missed by the Stage 1 Survey of the present study. The agestandardized prevalence rate of dementia estimated by the other large-scale urban studies in $\mathrm{Japan}^{6-8,12,13)}$ ranged between 4.0 and 5.0 per 100 persons, which is consistent with that in the present study.

The present results also supports the findings from other studies of dementia that the prevalence increases with aging in both sexes and that larger gender differences are found in the older age groups.

\section{Incidence}

In contrast to the abundance of reports on the prevalence of dementia, reports on incidence are limited ${ }^{19-28)}$. Several longitudinal studies are in progress $^{22,27,28,31)}$ and data on the incidence are being accumulated. A method for simulating the incidence has also been proposed ${ }^{37)}$. However, since most incidence studies have been limited in scale, it has been difficult to obtain precise estimates of the rates. Large-scaled studies such as the present one will make it possible to obtain more precise estimates including age-specific rates.

A methodological problem in estimating incidence of dementia based on longitudinal study is losses to follow-up. The incidence rates reported here are likely to be underestimated, as the rates in the present study were calculated for those living at home in 1988 and still living in Sendai City in 1991. Reduced life expectancy among the demented elderly ${ }^{25,38,39)}$ suggests that there would be higher incidence among the decedents. Also, those staying at long-term care facilities may have higher incidence of dementia. Several studies in the past have sought information for deceased persons. In the Beijing study ${ }^{26)}$, information from an informant was used. But, the accuracy of information by lay informants is questionable. Another source of information is death certificates or medical charts ${ }^{28)}$. In order to address this issue, we obtained all death certificates. Among 48 deceased subjects who were demented in 1988, only two (4.2\%) were described as having dementia on their death certificates. Thus, we concluded that the death certificate was not a useful source for ascertaining dementia. Accordingly, we decided to estimate the incidence based on survivors. However, as the number of the study sample was large and the follow-up period was only three years, the effects of losses to follow-up on the estimation of incidence seems not serious. It is unlikely that there would be great difference between our figure and true incidence.

There have been three reports on the incidence of dementia in Japan; Tokyo Metropolitan ${ }^{20)}$, Miki Town ${ }^{22)}$, and a small island of Okinawa ${ }^{21}$. Incidence in Tokyo was derived from the cross-sectional study in $1980(\mathrm{~N}=4,520)$, by asking the time of onset of dementia symptoms to the proxies of the demented elderly. The cases whose symptoms appeared within 12 months before the survey were regarded as incident ones, and overall incidence rate per year among those aged 65 and over was estimated as 1.1 per 100 persons $^{20)}$.

Other two studies were based on a longitudinal design, and both of them dealt with rural community. A study on a small isolated island of Okinawa was based on one-year follow-up on 263 persons aged 65 and over in 1984, and the overall incidence was estimated as $1.14^{21)}$. A study on Miki Town was a yearly follow-up during 1987-1988 on residents aged 65 years and over $(\mathrm{N}=3,754)$, and is still on-going ${ }^{22)}$. The 
age-standardized incidence rate in Miki Town calculated using age-specific rates in the report, 0.92 per 100 persons, was lower than that in Sendai City. The instruments and diagnostic procedure in their study are similar to ours. It is possible that there is true difference in the incidence rate of dementia between rural and urban areas, since the age-standardized prevalence rate in Miki Town, 3.7 per 100 persons, was also lower than that in Sendai City. However, the incidence rate for these two rural studies may not be stable, since it was derived from a yearly follow-up study. A clearer picture of urban-rural differences in the incidence of dementia will be availavle when additional follow-up is completed in Miki Town.

Data on the incidence are very sparse. To our knowledge, there are only two large-scale longitudinal studies on dementia in Japan; Miki Town and Sendai City. Further studies are required to elucidate the incidence and the factors which affect the course of dementia.

\section{ACKNOWLEDGMENTS}

The second author (I.T.) was supported in part by Uehara Memorial Foundation, Tokyo, Japan, during his stay at the Johns Hopkins University.

The authors thank Ms. H. Ito and public health nurses at Sendai City Health Department.

\section{REFERENCES}

1. United Nations. World population prospects 1990. New York, NY: United Nations, 1991. (Population studies no. 120).

2. Institute of Population Problems Ministry of Health and Welfare. Selected demographic indicators from the United Nations popoulation projections as assessed in 1990. Tokyo, Japan: Institute of Population Problems, Ministry of Health and Welfare, 1991. (Research series, no. 267) (in Japanese).

3. Ozaki T, Aoki S, Sasaki Y, et al. Health and medical services for the elderly. J Health Welfare Stat, 1991; 38(14): 151-166 (in Japanese).

4. Maeda $H$. The simulation study for planning medical health and welfare services. J Health Welfare Stat, 1992 ; 39(4) : 22-33 (in Japanese).

5. Division of Welfare, Tokyo Metropolitan Government. Life style and health problems in elderly in 1980. Tokyo, Japan : Division of Welfare, Tokyo Metropolitan Government, 1981 (in Japanese).

6. Karasawa A, Kawashima K, Kasahara H. Epidemiological study of the senile in Tokyo Metropolitan Area. Proceedings of World Psychiatric Association Regional Symposium. Kyoto, Japan : World Psychiatric Association, 1982 : 285-289.

7. Yokoi $S$, Nakae $S$. Investigation of prevalence of dementia among aged people domiciled in Yokohama City. Jpn J Clin Psychiatry, 1983; 12:1541-1548 (in
Japanese).

8. Hasegawa K, Homma A, Sato $H$, et al. An epidemiological study of age-related dementia in KanagawaKen. Gerontopsychiatry, 1984 ; 1 : 94-105 (in Japanese).

9. Division of Welfare, Kawasaki City Government. The report of epidemiological survey for elderly in Kawasaki City. Kawasaki, Japan: Division of Welfare, Kawasaki City Government, 1985 (in Japanese).

10. Hasegawa K, Homma A, Imai Y. An epidemiological study of age related dementia in the community. Int $J$ Geriatr Psychiatry, 1986; 1: 45-55.

11. Shibayama H, Kasahara $\mathrm{Y}$, Kobayashi $\mathrm{H}$, et al. Prevalence of dementia in a Japanese elderly population. Acta Psychiatr Scand, 1986; 74 : 144-151.

12. Division of Welfare, Tokyo Metropolitan Government. Life style and health problems in elderly in 1988. Tokyo, Japan: Division of Welfare, Tokyo Metropolitan Government, 1990 (in Japanese).

13. Division of Welfare, Aichi Prefecture Government. The Report of epidemiological survey for elderly in Aichi prefecture. Nagoya, Japan: Division of Welfare, Aichi Prefecture Government, 1991 (in Japanese).

14. Weissman MM, Myers JK, Tischler GL, et al. Psychiatric disorders (DSM-III) and cognitive impairment among the elderly in a U.S. urban community. Acta Psychiatr Scand, $1985 ; 71: 366-379$.

15. Jorm AF, Korten AE, Henderson AS. The prevalence of dementia: A quantitative integration of the literature. Acta Psychiatr Scand, 1987; $76: 465-479$.

16. Li G, Shen YC, Chen $\mathrm{CH}$, et al. An epidemiological survey of age-related dementia in an urban area of Beijing. Acta Psychiatr Scand, 1989; $79: 557-563$.

17. Robertson D, Rockwood K, Stolee P. The prevalence of cognitive impairment in an elderly Canadian population. Acta Psychiatr Scand, 1989 ; 80 : 303-309.

18. Livingston G, Hawkins A, Graham N, Blizard B, Mann, A. The Gospel Oak Study: prevalence rates of dementia, depression and activity limitation among elderly residents in Inner London. Psychol Med, 1990; 20 : 137-146.

19. Copeland JRM, Dewey ME, Davidson IA, Saunders PA, Scott A. Geriatric mental state-AGECAT: Prevalence, incidence and long-term outcome of dementia and organic disorders in the Liverpool study of continuing health in the community. Neuroepidemiology, 1992; 11(suppl 1): 84-87.

20. Karasawa A. Epidemiology of dementia in the elderly. In: Hasegawa $\mathrm{K}$ ed. Dementia in the elderly. Tokyo, Japan: Kanehara \& Co. Ltd.,1984: 99-112 (in Japanese).

21. Ichinowatari $\mathrm{N}$, Makiya $\mathrm{H}$, Doi $\mathrm{M}$, Tatsunuma, $\mathrm{T}$. Epidemiological investigation of old age mental disorders in a small isolated island of Okinawa Prefecture ; Comparison between mental disorders observed in 1979 and 1984. J Geriatr Psychiatry, 1988; 90: 612-635 (in Japanese).

22. Fukunishi I, Hayabara T, Hosokawa K. Epidemiological surveys of senile dementia in Japan. Int J Soc Psychiatry, $1991 ; 37: 51-56$.

23. Nilsson LV. Incidence of severe dementia in an urban sample followed from 70 to 79 years of age. Acta Psychiatr Scand, 1984 ; 70 : 478-486.

24. Sayetta RB. Rates of senile dementia-Alzheimer's type in the Baltimore longitudinal study. J Chron Dis, 1986; 39 : 271-286.

25. Aronson MK, Ooi WL, Geva DL, et al. Dementia: Age-dependent incidence, prevalence, and mortality in the old old. Arch Intern Med, 1991; 151: 989-992. 
26. Li G, Shen YC, Chen $\mathrm{CH}$, et al. A three-year follow-up study of age-related dementia in an urban area of Beijing. Acta Psychiatr Scand, 1991; 83 : 99-104.

27. Mann AH, Livingston G, Boothby $H$, Blizard, R. The Gospel Oak study: The prevalence and incidence of dementia in an inner city area of London. Neuroepidemiology, 1992; 11(suppl 1): 76-79.

28. Morgan K, Lilley J, Arie T, et al. Incidence of dementia : Preliminary findings from the Nottingham longitudinal study of activity and aging. Neuroepidemiology, 1992; 11(suppl 1): 80-83.

29. Gauthier S, McDowell I, Hill G. Canadian study of health and aging (CaSHA). Psychiatr J Univ Ottawa, 1990; 15: 227-229.

30. Andrews GR. The longitudinal study of aging-The place of multidimensional population based cross national studies. Proceedings of WHO International Workshop: Well-being of the elderly. Sendai, Japan: WHO International Workshop, 1991: 57-60.

31. Amaducci L. Italian Iongitudinal study on aging: Incidence study of dementia. Neuroepidemiology, 1992 ; 11(suppl 1) : 19-22.

32. Division of Health Services for the Aged, Ministry of Health Welfare. Manual for caring demented elderly. Tokyo, Japan: Japan Public Health Society, 1991 (in
Japanese).

33. Hasegawa K. The clinical issues of age-related dementia. Tohoku J Exp Med, 1990; 161(suppl) : 29-38.

34. Folstein MF, Folstein SE, McHugh PR. Mini-Mental State: A practical method for grading the cognitive state of patients for the clinician. J Psychiatr Res, 1975; 12: 189-198.

35. The American Psychiatric Association. Diagnostic and Statistical Manual of Mental Disorders. 3rd edn-revised. Washington, DC: American Psychiatric Association, 1987.

36. Abe Y, Onodera M, Kamiie K, Saito F, Matsuei T. The revised Japan standard population. J Health Welfare Stat, 1991; 38(5): 12-16.

37. Dewey M. Estimating the incidence of dementia in the community from prevalence and mortality results. Int $\mathbf{J}$ Epidemiol, 1992 ; 21 : 533-536.

38. Campbell AJ, Diep C, Reinken J, McCosh L. Factors predicting mortality in a total population sample of the elderly. J Epidemiol Community Health, 1985; 39 : 337-342.

39. Jagger C, Clarke M. Mortality risks in the elderly: Five-year follow-up of a total population. Int $J$ Epidemiol, 1988; 17: 111-114. 